\title{
Preface KSE 2016
}

The 8th International Conference on Knowledge and Systems Engineering (KSE 2016) was held in Hanoi, Vietnam from the $6^{\text {th }}$ to $8^{\text {th }}$ October 2016. This volume contains extended and revised versions of the best articles presented at KSE 2016 . These articles were selected by members of the organizing and scientific committee, and evaluated based on their contents, innovations and contributions as well as the oral presentation during the conference. The extended and revised articles were reviewed by at least two independent reviews, in a blind review process.

The main theme, emerging from the articles in this volume, concerns the manipulation and transformation of data into information, and knowledge acquisition and management. The authors analyzed data from various sources and types, including unstructured textual data and data on currency exchange rates and transportation systems.

The first article in this volume, 'Exploring Alignment-Classification Methods in the Context of Professional Writing Assistance' by Mai Duong, Minh-Quoc Nghiem and Ngan Luu-Thuy Nguyen, propose a method, based on word alignment and classification, which learns from corrections made to a text. Such a method is expected to play a pivotal role in language learning. The authors also propose a novel taxonomy for tagging learning corpora.

The next article, 'Automatically Classifying source code using Tree-based Approaches' by Anh Viet Phan, Phuong Ngoc Chau, Minh Le Nguyen and Lam Thu Bui focuses on one largely overlooked type of text data, namely source-code. They develop an algorithm for automatically classifying source-codes into corresponding labels. Their approach is expected to alleviate many of the drawbacks of classical software metrics.

In 'Learning Multiple Layers of Knowledge Representation for Aspect Based Sentiment Analysis', Duc-Hong Pham and Anh-Cuong Le address the issue of aspect-level sentiment analysis, and posit that the overall sentiment of a review is a function of the sentiment expressed per product-aspect. They perform sentiment analysis on hotel reviews using a multiple layer architecture.

The fourth article in this volume, 'A novel evolutionary multi-objective ensemble learning approach for forecasting currency exchange rates', by Lam Thu Bui, Van Truong Vu and Thi Thu Huong Dinh presents a technique for forecasting currency exchange rates. To fully capture the financial characteristics inherent in foreign exchange rate forecasting, the authors propose an ensemble method.

Finally, in 'A time-dependent model with speed windows for share-a-ride problems: A case study for Tokyo transportation', Phan-Thuan Do, Nguyen Viet Dung Nghiem, Ngoc-Quang Nguyen, Quang-Dung Pham, address the pertinent issue of transportation management in big cities. To this aim, they propose a fully time-dependent model of a public transport system allowing a taxi sharing system between passengers and parcels, with time constraints consideration. They analyze their model based on various factors, including the total benefit, the accumulating traveling time during the day, the number of used taxis and the number of shared requests.

Editors

Ashwin Ittoo

HEC Liège, Liège University, Belgium

Nguyen Le Minh Japan Advanced Institute of Science and Technology, Japan

Satoshi Tojo Japan Advanced Institute of Science and Technology, Japan 